\title{
Algorithms for analysis of stability and dynamic characteristics of signal generators at the physical level in FANET networks
}

\author{
Gleb S. Vasilyev 1,*, Oleg R. Kuzichkin ${ }^{1}$, Dmitriy I. Surzhik ${ }^{2}$ and Igor A. Kurilov² \\ ${ }^{1}$ Belgorod National Research University, 85 Pobedy St., 308015 Belgorod, Russia \\ ${ }^{2}$ Vladimir State University, 87 Gorky St., 600000, Vladimir, Russia
}

Keywords: Unmanned aerial vehicle, UAV, Flying ad-hoc network, FANET, Physical level, Stability, Dynamic characteristic.

\begin{abstract}
To ensure the reliability of the physical level of Flying Ad Hoc Networks (FANET), the article substantiates the relevance of the study of stability and dynamic processes of signal formation and processing paths in the terminal and intermediate network equipment. The limitations of the known methods of the theory of automatic control, which make it difficult to perform the analysis of essentially nonlinear high-order signal generators, are shown. To analyze the stability of such formations proposed algorithm based on the use of the Nyquist criterion and approximation of the characteristic polynomial of a device by continuous piecewise functions; to analyze the dynamic characteristics of the algorithm based on spectral method of the input signal approximation and the frequency characteristics of a device by continuous piecewise functions.
\end{abstract}

\section{Introduction}

Organization of information exchange in Flying Ad Hoc Networks (FANET) requires creation of effective interaction between individual unmanned aerial vehicles (UAVs) (UAVUAV channel), as well as UAV and ground control centers (UAV-ground station channel) [1]. The process of information transmission in FANET networks is regulated by protocols of different levels, and the main differences from the classical protocols of hierarchical networks are typical for the four lower levels: physical [2-4], channel [4,5], network and transport $[6,7]$. Building a reliable network requires competent decisions at the physical level (choosing the type of modulation and coding, choosing the structure of antennas). Moreover, the task of designing and analyzing the physical layer of FANET networks in comparison with other types of ad-hoc networks (sensor, vehicular - VANET, etc.) is further complicated by a number of factors [8]:

- significantly increased range of the communication channel, which is why the quality of the connection is more affected by the conditions of propagation of the radio signal;

\footnotetext{
*Corresponding author: vasilievgleb@yandex.ru
} 
- extremely high node mobility.

To solve these problems when building a network, it is necessary to use adequate models of communication channel [2-4] and node mobility [9,10]. Unfortunately, the known models of the physical layer of FANET networks, as a rule, do not take into account the impact of external conditions on the operation of communication equipment: weather, natural interference and the influence of electronic warfare, own noise of electronic cascades, etc. The negative impact of external destabilizing factors can cause [11]:

- reduction of signal-to-noise ratio due to attenuation of useful signal or increase of noise level;

- problems with synchronization of transmitting and receiving nodes due to jitter growth;

- the phenomenon of intersymbol interference due to the limited bandwidth of the communication channel and signal processing paths;

- self-excitation of the paths of the transmitting and receiving module of the network.

The study of these effects requires modeling and analysis of the stability of signal generation and processing paths, as well as dynamic characteristics (transients) of these paths. The solution of these problems can be performed on the basis of known approaches of the theory of automatic control: algebraic and frequency stability criteria [12-17], methods of solving ordinary differential equations [18-21]. However, significant difficulties arise due to the high order and significant nonlinearity of the devices in question.

The aim of the work is to develop algorithms for analyzing the stability and dynamic characteristics of nonlinear high-order signal generators at the physical level in FANET networks.

\section{Main part}

\subsection{Approximation of the characteristics of the signal formation by piecewise continuous functions}

The authors' studies have shown the effectiveness of applying the approximation of various influences, nonlinear and frequency characteristics of radio devices, in particular, signal generators, continuous piecewise functions (CPF) with high accuracy and a simple analytical form [22-28].

At the moment, several types of CPF have been developed, the main of which are switching and including CPF shown on Fig. 1.

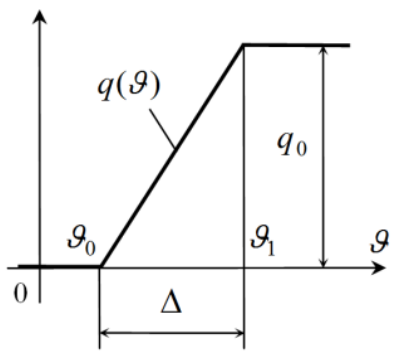

A)

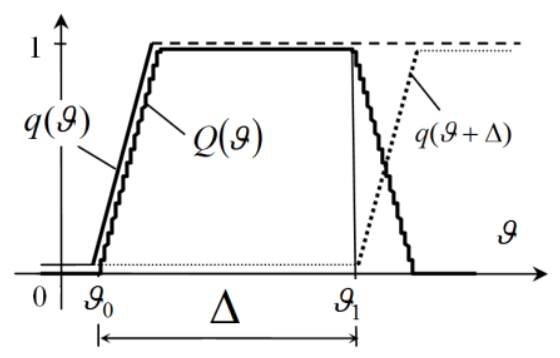

B)

Fig. 1. Switching (A) and including (B) CPF.

The switching CPF serves as a base for receiving other CPFs and consists of three linear sections. A large number of such approximating functions allows us to represent essentially nonlinear characteristics and effects with high accuracy. 
General expression of the approximating function based on the sum of the switching CPFs for the generalized variable is as follows

$$
q_{\Sigma}(\vartheta)=\sum_{\mathrm{i}=0}^{N-1} q_{i}(\vartheta)=\sum_{\mathrm{i}=0}^{N-1} \frac{q_{0_{i}}}{2 \Delta_{i}}\left(\left|\vartheta-\vartheta_{\mathrm{i}}\right|-\left|\vartheta-\vartheta_{\mathrm{i}}+\Delta_{i}\right|+\Delta_{i}\right)
$$

where $\vartheta_{i}$ is an argument value in the current approximation node, $N$ is a number of approximation nodes, $q_{0_{i}}$ is height of the $i$-th function (approximation coefficient), $\Delta_{i}=\vartheta_{\mathrm{i}+1}-\vartheta_{\mathrm{i}}$ is approximation step.

Another type of CPF considered (including) is an elementary approximating function with a plane vertex consisting of four linear modules and taking the value 1 in the interval $\left[\vartheta_{0} ; \vartheta_{l}\right.$ ] and 0 outside it. This allows a simple way to approximate a specific characteristic on a separate interval, where its value is 1 and ignore its change outside this interval.

General expression of the inclusive CPF $\vartheta$ is as follows

$$
Q_{\mathrm{i}}(\vartheta)=K_{\sigma} \sum_{\lambda=0}^{1} \sum_{\gamma=0}^{1}(-1)^{\lambda+\gamma}\left|\vartheta-\vartheta_{i}-\gamma \Delta_{\mathrm{i}}-\frac{\lambda}{2 K_{\sigma}}\right|,
$$

where $K_{\sigma}$ is the steepness of the CPF; $\lambda, \gamma$ are coefficients equal to 0 or 1 .

To approximate an arbitrary nonlinear characteristic (Fig. 2), it is required to multiply $\mathrm{N}$ inclusive CPFs by linear functions corresponding to the given plots:

$$
\mathrm{Q}_{\Sigma}(\vartheta)=\sum_{i=0}^{N-1} f_{i}(\vartheta)=\sum_{i=0}^{N-1}\left(K_{i} \vartheta+\mathrm{L}_{i}\right) Q_{i}(\vartheta)
$$

where $N$ is a maximum number of the approximation node, $K_{i}=\frac{f\left(\vartheta_{i+1}\right)-f\left(\vartheta_{i}\right)}{\Delta_{i}}$ is steepness of the $i$-th approximating line, $\mathrm{L}_{i}=f\left(\vartheta_{i}\right)-K_{i} \vartheta_{i}$ is a value of the $i$-th linear function at $\vartheta=0$.



Fig. 2. Approximation of an arbitrary nonlinear function by including CPFs.

Due to the obvious advantages, the considered CPFs can be effectively used to expand the scope of the known analytical methods of stability analysis and dynamic modes of various devices for signal formation and processing of terminal and intermediate network equipment with linear and nonlinear signal conversion. 


\subsection{Algorithm for analysis of parametric stability of signal generators}

To guarantee the absence of self-excitation of the paths of the transmitting and receiving module of the FANET network under the influence of various destabilizing factors, it is necessary to perform a stability analysis of these paths. In general, an arbitrary device is stable if all the roots of the characteristic polynomial of its transfer function have a negative real part. If this condition is met and there is at least one purely imaginary root, the device is at the stability boundary [12].

To solve the problem of stability analysis without finding roots, it is possible to use known approaches of the theory of automatic control, namely algebraic or frequency stability criteria [12-17]. The disadvantage of the known criteria is that they are focused on the stability analysis of the system with fixed parameter values. At the same time, deviation of the parameters of the elements during manufacture or operation can cause a loss of stability. To take this phenomenon into account, it is necessary to construct regions of stable values of system parameters, i.e. to investigate its parametric stability.

For the parametric stability analysis of the signals formation devices of the terminal and intermediate network equipment with linear and nonlinear transformation of signals the algorithm based on approximation by means of CPF and use of frequency Nyquist criterion is developed [12]. The block diagram of this algorithm is shown in Fig. 3.


Fig. 3. Block diagram of the stability analysis algorithm of signal generators based on approximation using $\mathrm{CPF}$ and the Nyquist frequency criterion.

The algorithm is based on the analysis of characteristic polynomials of the transfer function of the signal generator, determined by the presence of one or more feedback loops in the device scheme:

$$
1+N_{2} M_{2}(p)=0
$$

where $N_{2}$ is frequency-independent transmission coefficient of the open feedback loop, $M_{2}(p)$ is frequency-dependent transmission coefficient of the open feedback loop (usually a filter), $p=\mathrm{d} / \mathrm{d} t$ 
is a Laplace operator.

With an arbitrary configuration and order of the filter $M_{2}(p)$, its complex transmission coefficient in the frequency domain can be represented as

$$
M_{2}(\mathrm{j} \omega)=\frac{\sum_{i=0}^{I} \alpha_{i}(\mathrm{j} \omega)^{i}}{\sum_{i=0}^{I} \beta_{i}(\mathrm{j} \omega)^{i}},
$$

where $I$ is the filter order, $\alpha_{i}, \beta_{i}$ are the filter coefficients.

The former will be stable if the imaginary part of expression (5) is 0, i.e.

$$
\operatorname{Im}\left[M_{2}\left(\mathrm{j} \omega_{\mathrm{k}}\right)\right]=0
$$

where $\omega_{k}$ are critical frequencies (root values corresponding to the stability boundary), $\mathrm{k}$ is the root number.

Substituting the expression (5) in the stability condition (6) and highlighting the imaginary component of the complex transfer function, a general expression of the characteristic equation is obtained. To find the roots of this polynomial, the general solution of which is not produced marking his left side as a function of frequency $f(\omega)$ and approximation with CPF of the form (3), and the resulting roots of the polynomial are determined as points of intersection of the approximating straight lines with the abscissa axis.

According to expression (4), it is obvious that the boundary values of the frequencyindependent coefficient of the former $N_{2 k}$ for each true root correspond to the values

$$
N_{2 \mathrm{k}}=\mathrm{N}_{2}\left(\omega_{k}\right)=-\frac{1}{M_{2}\left(\mathrm{j} \omega_{k}\right)} .
$$

The signal former will be stable with any type and order of the used filter at value of frequencyindependent coefficient $N_{2}=0$. Thus, to find the resulting boundary of parametric stability, it is necessary to choose one negative and one positive nearest to zero from all the boundary coefficients (7), i.e. to determine the region $N_{2}^{\text {low }} \leq N_{2} \leq N_{2}^{u p}$. The lower bound of this area corresponds to the maximum of all negative $N_{2 \mathrm{k}}$ values, the upper bound - to the minimum of all positive $N_{2 \mathrm{k}}$ values.

Fractional-rational representation of the transfer coefficient (5) is not a prerequisite for the algorithm. In the case of analysis of pure delay links with a transcendental (exponential) transfer function, the value of the transfer coefficient of the feedback loop filter $M_{2}(p)$ at critical frequencies $\omega_{k}$ can be calculated directly, followed by checking the condition (6). When modeling the physical layer of FANET links of this type can be represented, for example, delay lines in the network equipment, as well as individual atmospheric channels with multipath reception (in the latter case, you need to take into account the attenuation). When using the algebraic Raus-Hurwitz stability criterion, it would be necessary to additionally perform a fractional-rational approximation of the transcendental transfer function. In addition, when using the developed algorithm, an increase of the device order under study does not cause a significant increase in computational costs, which would be inevitable due to a sharp increase in the number and dimension of the Hurwitz matrix minors. These factors determine effectiveness of the proposed algorithm. 


\subsection{Algorithm of dynamic characteristics evaluation of signal formers}

To assess the duration and properties of transients in signal generators, it is necessary to study their dynamic properties, considering the reaction to typical deterministic effects.

To calculate the dynamic characteristics of signal generators, an algorithm based on approximation by $\mathrm{CPF}$ and spectral method is developed. Its block diagram is shown in Fig. 4.

The first stage of the presented algorithm is approximation of the input signal acting on the former by the sum of switching CPFs (1), calculation of its spectrum $X(j \omega)$ using the Fourier transform and determination of the transmission coefficients of the structural units of the device. Further, with a known influence spectrum and the transfer function of the entire signal former $H(j \omega)$, it is required to find the output signal of the former $S_{\text {out }}(j \omega)$ from its spectrum by the inverse Fourier transform. For this purpose, using the spectral method and approximation of the real $S_{R}(\omega)=\operatorname{Re}\left[S_{\text {out }}(j \omega)\right]$ and imaginary $S_{I}(\omega)=\operatorname{Im}\left[S_{\text {out }}(j \omega)\right]$ spectral density of the output signal of the device by switching CPFs, generalized analytical expressions for this calculation are obtained. In the case of the real spectrum $S_{R}(\omega)$ approximation from the logarithmic frequency expression has the form

$$
y(t)=x(t) \cdot H(0)+\frac{2}{\pi t} \sum_{i=0}^{N-1} \frac{a_{0 i}}{\Delta_{\lambda}}\left[S i\left(\omega_{i+1} t\right)-S i\left(\omega_{i} t\right)\right]
$$

where $x(t)$ is an input signal of the device; $H(0)$ - transfer function value at zero frequency; $a_{0 i}=S_{R}\left(\omega_{\mathrm{i}}\right)-S_{R}\left(\omega_{\mathrm{i}+1}\right)$ is a coefficient of CPF approximating $S_{R}(\omega)$ at the current segment $\left[\omega_{\mathrm{i}} ; \omega_{\mathrm{i}+1}\right] ; \Delta_{\lambda}$ is a step of logarithmic frequency; - $\operatorname{Si}(x)$ is integral sine.

The algorithm controls accuracy of the calculated dynamic characteristics of the signal generator based on the comparison of the achieved (RMSreal) and permissible (RMSallow) RMS error. If the device under study is essentially nonlinear, it is advisable to first perform a piecewise linear approximation of the characteristics of the nonlinear links, and then use the algorithm (Fig. 4) for individual linear sections, taking into account the initial conditions for each current section, and perform crosslinking of the obtained partial solutions.

\section{Conclusion}

The task of modeling FANET networks at the physical level has a significant complexity due to the extremely high mobility of nodes, as well as the strong influence of destabilizing factors on the propagation of radio signals due to the large length of communication channels between nodes. There is also a significant negative impact of interference of various kinds on the circuit paths of signal formation and processing as part of the transmitting and receiving modules of network nodes (especially in the operation of electronic warfare). The study of these effects requires modeling and analysis of the stability of signal generation and processing paths, as well as dynamic characteristics (transients) of these paths. It is shown that the high order and significant nonlinearity of the considered devices make it difficult to study the known methods of the theory of automatic control. An algorithm based on the Nyquist criterion and approximation of the characteristic polynomial of the device by continuous piecewise functions is proposed to analyze the stability of essentially nonlinear high-order formers. For the analysis of dynamic characteristics, an algorithm based on the spectral method and approximations of the input signal and the frequency response of the device by continuous piecewise functions is proposed. 


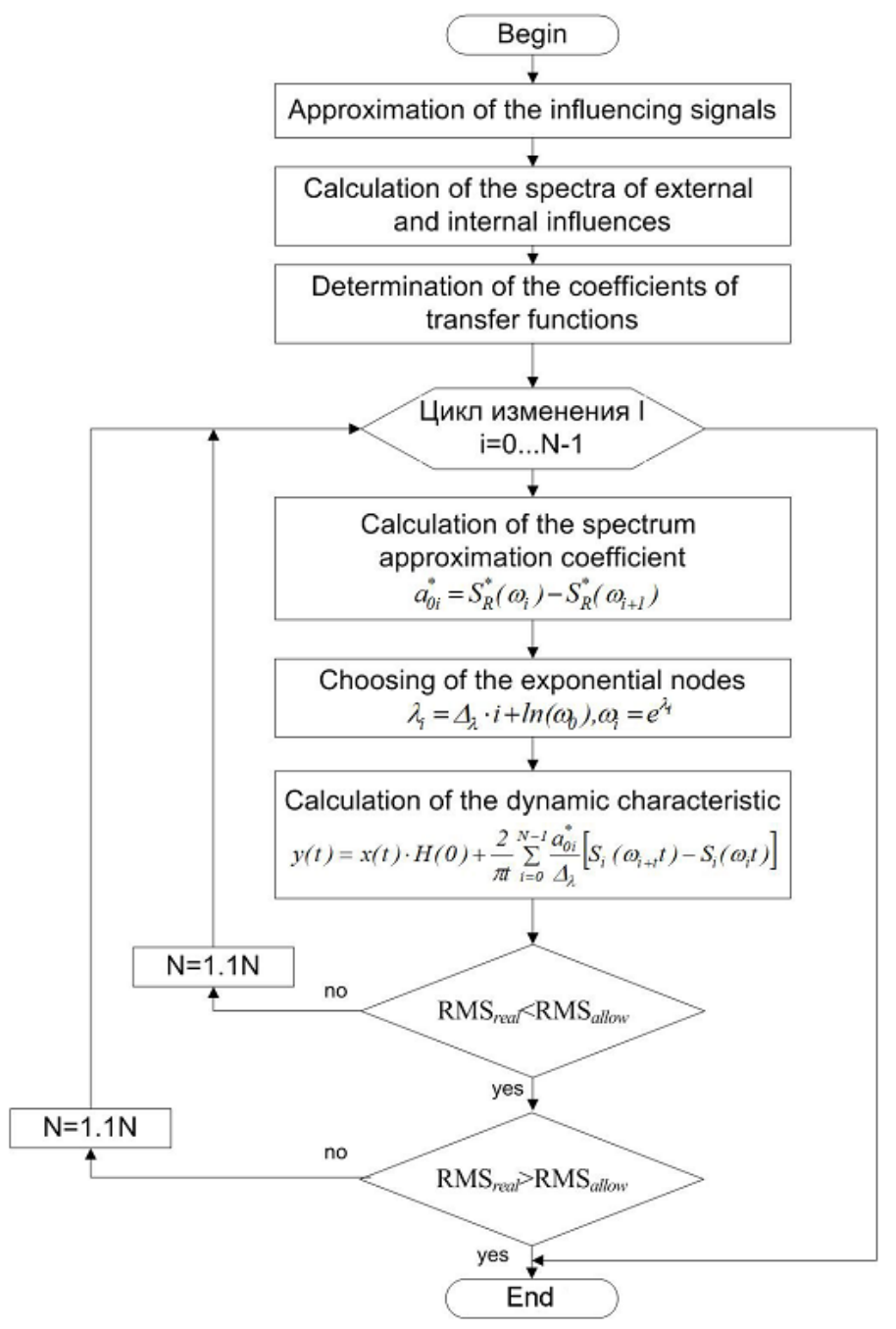

Fig. 4. Block diagram of the calculation algorithm of dynamic characteristics of signal generators, based on approximation by CPF and spectral method.

The work was supported by RFBR grant 19-29-06030-MK "Research and development of wireless adhoc network technology between UAVs and control centers of "smart city" on the basis of adaptation of transmission mode parameters at different levels of network interaction"

\section{References}

1. R. Austin. Unmanned aircraft systems: UAVS Design, Development and Deployment / John Wiley \& Sons, Ltd., 2010.

2. J. Yin, G. Holl, T. Elbatt, F. Bai, H. Krishnan, DSRC channel fading analysis from empirical measurement, in: Proceedings of the 1st IEEE International Workshop on Vehicle Communications and Applications (Vehiclecomm), 2006, pp. 25-27. 
3. I.Y. Abualhaol, M.M. Matalgah, Performance analysis of cooperative multi-carrier relay-based UAV networks over generalized fading channels, International Journal of Communication Systems 24 (8) (2011) 1049-1064.

4. O. Bazan, M. Jaseemuddin, On the design of opportunistic MAC protocols for multihop wireless networks with beamforming antennas, IEEE Transactions on Mobile Computing 10 (3) (2011) 305-319.

5. Z. Huang, C.-C. Shen, A comparison study of omnidirectional and directional MAC protocols for ad hoc networks, in: Global Telecommunications Conference, GLOBECOM, IEEE, 2002.

6. Karp, Brad \& Kung, Hsiang. (2000). GPSR: Greedy Perimeter Stateless Routing for Wireless Networks. Proceedings of the Annual International Conference on Mobile Computing and Networking, MOBICOM. 10.1145/345910.345953.

7. A. I. Alshabtat, L. Dong, J. Li, F. Yang, Low latency routing algorithm for unmanned aerial vehicles ad-hoc networks, International Journal of Electrical and Computer Engineering, 2010, Vol. 6, № 1, pp. 48-54.

8. Abuarqoub, Abdelrahman \& Hammoudeh, Mohammad \& Alfayez, Fayez \& Aldabbas, Omar. (2016). A Survey on Wireless Sensor Networks Simulation Tools and Testbeds. In book: Sensors, Transducers, Signal Conditioning and Wireless Sensors Networks Advances in Sensors: Reviews, Vol. 3 Chapter: 14 Publisher: IFSA.

9. E. Kuiper, S. Nadjm-Tehrani, Mobility models for UAV group reconnaissance applications, in: Proceedings of International Conference on Wireless and Mobile Communications, IEEE Computer Society, 2006, p. 33

10. W. Wang, X. Guan, B. Wang, Y. Wang, A novel mobility model based on semi-random circular movement in mobile ad hoc networks, Information Science 180 (3) (2010) 399413.

11. Sklar B.. Digital communication: theoretical foundations and practical applications / second edition / Prentice Hall PTR, 2002.

12. Besekersky.L., Popov E. Theory of automatic control systems. - SPb.: Profession, 2003. - 752 p.

13. Postnikov M. M. Stable polynomials. - Moscow: Nauka, 1981. - 176 p.

14. Neymark Yu. I. Dynamic systems and controlled processes. - Moscow.: Science, 1978.

15. Polyak, B. T. and Shcherbakov, P. S. Technique of D-decomposition solving linear matrix inequalities / / Automatics and telemechanics. - 2006. - No. 11. - P. 159-174.

16. Gryazina. E.N. To the theory of D-partition / / Automatics and telemechanics. - 2004. No. 12. - P. 15-28.

17. Gryazina E. N., Polyak B. T., Tremba A. A. The current state of the D-partition method. - Automatics and telemechanics. - 2008. - No. 12,. - P. 3-40.

18. Popov V.M. Hyperstability of automatic systems. - Moscow: Science, 1970. - 456 p.

19. Ditkin V.A., Prudnikov A. P. Integral transformations and operational calculus. Moscow.: Fizmatgiz, 1961. - 524 p.

20. Ditkin V.A., Prudnikov A. P. Handbook of operational calculus. - Moscow: High school, 1965. - P. 240.

21. Dech G. Guide to the practical application of the Laplace transform and the z-transform: translated from German. - Moscow: Science, 1971. - 288 p.

22. Shostak R. I. Operational calculus. Study guide for technical colleges. Ed. 2nd, Moscow: High school, 1972. - 280 p. 
23. Surzhik, D.I. Correlation Analysis of the PLL with an Arbitrary Filter Based on Continuous Piecewise-Linear Functions. / D.I. Surzhik, G.S. Vasilyev // 2013 International Siberian Conference on Control and Communications (SIBCON). Proceedings. - Krasnoyarsk: Siberian Federal University. Russia, Krasnoyarsk, September 12-13, 2013. IEEE Catalog Number: CFP13794-CDR SCOPUS, IEEEXplore, http://conf.sfu-kras.ru/conf/sibcon ISBN: 978-1-4799-1060-1/13/2013 IEEE.

24. Kurilov, I.A. Methods of analysis of radio devices based on functional approximation / Kurilov, V.V. Romashov, E.A. Zhiganov, D.N. Romanov, G.S. Vasiliev, S.M. Kharchuk, D.I. Surzhik // Radio engineering and telecommunication systems. - 2014. - No. 1. - P. 35-49.

25. Vasiliev G. S. Study of signal converter stability based on continuous piecewise linear functions / I. A. Kurilov, G. S. Vasiliev, S. M. Kharchuk, D. I. Surzhik / / Radio engineering and telecommunication systems. No. 1, 2012. Pp. 4-7.

26. Vasiliev G. S. Analysis of dynamic characteristics of signal converters based on continuous piecewise linear functions / I. A. Kurilov, G. S. Vasiliev, S. M. Kharchuk / / Scientific and technical Bulletin of the Volga region, 2010. - No. 1. Pp. 100-104.

27. Kurilov, I.A. Research of static characteristics of converters of signals with a nonlinear control device / I.A. Kurilov, G.S. Vasilyev, S.M. Kharchuk, D.I. Surzhik // 2011 International Siberian Conference on Control and Communications (SIBCON)/ Proceedings. - Krasnoyarsk: Siberian Federal University. Russia, Krasnoyarsk, September 15-16, 2011. - p. 93 - 96. IEEE Catalog Number: CFP13794-CDR, IEEEXplore, http://conf.sfu-kras.ru/conf/sibcon ISBN: 978-1-4577-1069-8/11/2011 IEEE.

28. Vasilyev, G.S. Analysis of dynamic characteristics of the nonlinear amplitude-phase converter at complex input influence / G.S. Vasilyev, I.A. Kurilov, S.M. Kharchuk, D.I. Surzhik // 2013 International Siberian Conference on Control and Communications, SIBCON 2013. - Catalog Number: CFP13794-CDR. SCOPUS, IEEEXplore, ISBN: 978-1-4799-1062-5/13/2013 IEEE. - $\quad$ p. 6693641. DOI: 10.1109/SIBCON.2013.6693641.

29. Vasilyev, G.S. Analysis of parametrical stability of the amplitude-phase converter with various filters / G.S. Vasilyev, I.A. Kurilov, S.M. Kharchuk // 2013 International Siberian Conference on Control and Communications, SIBCON 2013. - IEEE Catalog Number: CFP13794-CDR SCOPUS, IEEEXplore, ISBN: 978-1-4799-1062-5/13/2013 IEEE. - p. 6693640. DOI: 10.1109/SIBCON.2013.6693640. 